\title{
A Study from the Perspective of Nature-Inspired Metaheuristic Optimization Algorithms
}

\author{
Deepthi S \\ Dept. of Computer Science and Engineering \\ Mar Baselios College of Engineering and \\ Technology \\ Thiruvananthapuram, India
}

\author{
Aswathy Ravikumar \\ Dept. of Computer Science and Engineering \\ Mar Baselios College of Engineering and \\ Technology \\ Thiruvananthapuram, India
}

\begin{abstract}
There are various metaheuristic algorithms which can be used to solve optimization problems efficiently. Among these algorithms, nature-inspired optimization algorithms are attractive because of their better results. In this paper, four types of metaheuristic algorithms such as ant colony optimization algorithm, firefly algorithm, bat algorithm and cuckoo search algorithms were used as the basis for comparison. Ant colony optimization algorithm is based on the interactions between social insect, ants. Firefly algorithm is influenced by the flashing behavior of swarming firefly. Cuckoo search uses brooding parasitism of cuckoo species and bat algorithm is inspired by the echolocation of foraging bats.
\end{abstract}

\section{Keywords}

Metaheuristic, ant colony optimization algorithm, firefly algorithm, bat algorithm, cuckoo search algorithm.

\section{INTRODUCTION}

Optimization is a field of science in which the exploration of best values of parameters of the problem are done under specified conditions i.e. generally it will target to find parameter values in particular which enable an objective function to generate the maximum or minimum value. Problems in optimization can be categorized into different categories like Metaheuristic, Combinatorial optimization, Linear programming, Integer programming [1]. In optimization of a design, the design objective could be simply to minimize the cost of production or to maximize the efficiency of production. An optimization algorithm is a procedure which is executed iteratively by comparing various solutions till an optimum or a satisfactory solution is found.

A number of nature-inspired optimization algorithms have been emerged recently with designs that imitate the swarm behavior. Even if each of the individual search agents searches for its path in its own way, they swarm together collaboratively towards a common optimization goal. The nature-inspired optimization algorithms proposed to date have gained much attention among computer science researchers [7].

The nature inspired algorithm acts as a domain for swarm intelligence (SI) based algorithms and bio-inspired algorithms. Swarm intelligence has characteristics such as self-organization, co-evolution and learning during iteration process. Using this algorithm, large scale optimization is possible by parallelizing multiple agents. SI Algorithms can again be classified as trajectory based and population based (e.g. ant colony optimization algorithm, bat algorithm) depending on the trajectory of search path. Based on interaction to multiple agents, algorithm is classified as attraction (e.g. firefly algorithm) based and non-attraction based and finally based on updating equations, classification is done as rule based and equation based (e.g. cuckoo search) [6].

Metaheuristic algorithms are considered to be the most efficient algorithms to solve optimization problems as compared to other algorithms because of their easy implementation and accurate result production [3]. Every metaheuristic algorithm has mainly two components. First one is exploitation (intensification) and other one is exploration (diversification) [8]. Exploration means focus search procedure to a local region by exploiting information whatever is available in current location in that region, while exploration means explore the search space on global level in order to generate diverse solutions[9],[10].

In this paper our aim is to compare the firefly algorithm with bat algorithm, ant colony optimization algorithm and cuckoo search on factor like run time and success rate. Rest of the paper is organized as follows. The Ant Colony optimization, Firefly, Cuckoo search and Bat algorithms are briefly explained in Section 2. Comparison of these algorithms is done in Section 3. Section 4 finally concludes the paper.

\section{ALGORITHMS}

Firefly Algorithm (FA), Ant Colony optimization (ACO), Bat Algorithm (BA) and Cuckoo Search (CS) are named so because of the algorithm's resemblance with these social insects.

\subsection{Firefly Algorithm (FA)}

Firefly Algorithm is a bio-inspired metaheuristic algorithm. It is based on the motion and behavior of fireflies [2]. This algorithm is formulated based on three idealized rules:

- $\quad$ All fireflies are considered to be unisex. So each one will get attracted to other irrespective of sex.

- Attraction is directly proportional to the brightness of fire firefly. For any two flashing fireflies, the less bright one will move towards the brighter one. As the distance between fireflies increases, brightness gets decreased and eventually attractiveness too. If no fireflies are present throughout the vicinity, then it will move around randomly.

- Brightness of firefly is associated with an objective function. In case of maximization problem, brightness is proportional to value of objective function [2]-[3]. 
Algorithm 1 Firefly algorithm [3]

Objective Function $\mathrm{f}(\mathrm{X}), \mathrm{X}=\left(x_{1}, x_{2}, x_{3}, x_{4} \ldots x_{d}\right)$

Generate the initial population of $\mathrm{n}$ fireflies, $\mathrm{X}_{\mathrm{i}}, \mathrm{i}=$ $1,2, \ldots, \mathrm{n}$

Light intensity $I_{i}$ at $X_{i}$ is determined by $f\left(X_{i}\right)$

Define the light absorption coefficient $\gamma$

While $(\mathrm{t}<$ MaxGeneration)

$$
\begin{aligned}
& \text { For } \mathrm{i}=1: n, \text { all } \mathrm{n} \text { fireflies } \\
& \text { For } \mathrm{j}=1: n, \text { all } \mathrm{n} \text { fireflies (inner loop) } \\
& \quad \text { if }\left(I_{i}<I_{j}\right), \text { Move firefly } i \text { towards } j ;
\end{aligned}
$$

End if

Vary attractiveness with distance $i$ via $\exp \left[-\gamma^{\mathrm{r} 2}\right]$

End for $j$

End for $i$

Rank the fireflies and find the current global best solution $g^{*}$

\section{End while}

Post-process the results

\subsection{Ant Colony Optimization (ACO)}

Ant Colony Optimization algorithm finds optimal path by using positive feedback principle of pheromone. At the initial stage, none of the path have pheromone concentration. So ant will choose path randomly to move and leaves some amount of pheromone throughout the path they have followed. At this stage each path contains uneven concentration of pheromone. Now ant can choose path based on the concentration of pheromone. Normally ant will choose path which is having highest concentration. Upon selecting a path, ant will increase the pheromone concentration in the selected path during its journey.

Higher the concentration of pheromone in a path, higher the probability with which ant select the path and so the concentration in that particular path get strengthened. At last, all ants will join at the path that is having highest concentration level and it finds the optimal path [4].

Algorithm 2 Ant Colony Optimization Algorithm Create construction graph

Initialize pheromone values

While not stop-condition do

Create all ants' solutions

Perform local search

Update pheromone values

End while

\subsection{Bat Algorithm (BA)}

Bat-inspired algorithm is a metaheuristic search optimization algorithm. This algorithm is based on the echolocation behavior of bats with loudness and varying pulse emission [3].

The idealization of echolocation can be summarized as follows:
- Bat use echolocation to sense the distance and they have some magical mechanism to differentiate between prey and barrier.

To search prey, they fly randomly with velocity vi, at position $\mathrm{x}_{\mathrm{i}}$ with fixed frequency $\mathrm{f}_{\min }$, loudness $\mathrm{A}_{0}$ and wavelength $\lambda$.

- Loudness varies in different way between $\mathrm{A}_{0}$ (maximum value) to Amin (minimum value) Frequency ranges between $f_{\min }$ and $f_{\max }$. Similarly wavelength ranges between $\lambda_{\min }$ and $\lambda_{\max }$.

When bats start to search for food or prey, they will produce pulses with maximum loudness. As they approaches to prey, the loudness decreases. As soon as the bat find the prey, the loudness become the minimum possible value (usually zero), in order to avoid the loss of prey. Similarly, as the bat goes closer to the prey, the pulse emission rate gets increased. Once they find the prey, the pulse emission rate becomes the maximum possible value [11].

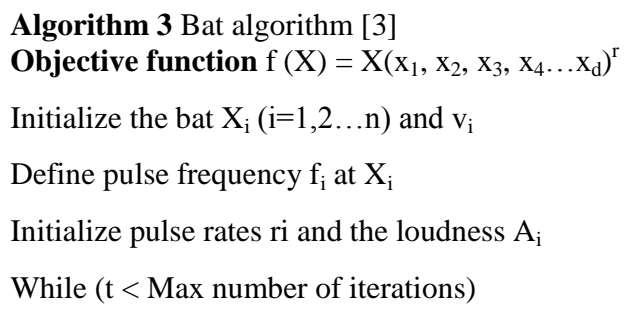

Generate new solutions by adjusting frequency, and updating velocities and locations/solutions

if $\left(\operatorname{rand}>r_{i}\right)$

Select a solution among the best solutions

Generate a local solution around the selected best solution

End if

Generate a new solution by flying randomly

if $\left(\operatorname{rand}<\mathrm{A}_{\mathrm{i}} \& \mathrm{f}\left(\mathrm{x}_{\mathrm{i}}\right)<\mathrm{f}\left(\mathrm{x}^{*}\right)\right)$

Accept the new solutions

Increase ri and reduce $\mathrm{A}_{\mathrm{i}}$

End if

Rank the bats and find the current best $\mathrm{x}^{*}$

End while

Post-process results and visualization

\subsection{Cuckoo Search (CS)}

The CS algorithm is a stochastic global search algorithm which is population based, in which pattern corresponds to a nest and each individual attribute of the pattern corresponds to egg of the bird [3]. Cuckoo Search is a Swarm Intelligence based, metaheuristic algorithm. This algorithm is based on the brooding characteristics of cuckoo bird. This algorithm can be used efficiently for the removal of noise from the network by training the network [12].Cuckoo lay egg in nest of other birds.

In CS method, egg of cuckoo is considered to be the solution for desired object function. In the simplest stage, each nest has only single egg. 
Cuckoo Search is based on three idealized rule:

- $\quad$ Each and every cuckoo lay one egg, which is used to represent a set of solution coordinates at a time and place it in a nest randomly.

- Those eggs which are considered to be the best, will be carried to next generation.

- There is a probability that a host bird can discover the alien egg. If such an event occurs, the host can either discard the egg or nest or both. This in turn will leads to building of new nest in a different location [11]

Algorithm 4 Cuckoo search [14]

Generate initial population of $\mathrm{N}$ host nest $\mathrm{x}_{\mathrm{i}} \forall \mathrm{i}, \mathrm{i}=1,2, \ldots, \mathrm{n}$

While ( $\mathrm{t}<\mathrm{M}$ axGeneration or (stop criterion))

do

Get a cuckoo randomly by Levy flights and

Evaluate itsfitness $\mathrm{F}_{\mathrm{i}}$.

Choose randomly a nest $\mathrm{j}$ among $\mathrm{N}$.

If $\left(F_{i}>F_{j}\right)$ then

Replace $\mathrm{j}$ by the new solution

End if

A fraction (pa) of worse nest are abandoned and new ones are built

Keep the best solutions (or nest with quality solutions).

Rank the solutions and find the current best.

End while

\section{ALGORITHM COMPARISON}

All the four types of algorithms are inspired by nature. They resemble some characteristics with the natural species. Characteristics and complexity of each one varies differently. Firefly algorithm is very powerful and it has a high speed of convergence. It perform local search efficiently. It has minimum running time and its success rate is better than others. Functional complexity had no effect on firefly algorithm. It requires more resources and CPU time.

Bat Algorithm had a disadvantage that, it does not memorize any history of better solution for each bat. It is unable to get rid of local search completely. It only needs less CPU time as compared to Firefly and Bat algorithms [2].

In Table.1, comparison of these four algorithms (Bat, Ant colony, Cuckoo and Firefly) are done by taking parameters running time, and success rate. Each algorithm will come under more than one classification. Basically all these algorithms are metaheuristic, Swarm Intelligence based algorithms. Cuckoo search is an equation based algorithm. All the others are population based algorithm. Firefly is also included in the category of attraction based algorithms because of their attraction towards other unisexual fireflies.।

Firefly Algorithm is based on the flashing behavior of swarming firefly. Ant colony optimization is based on the interaction between the groups of ant. Bat algorithm is based on the echolocation behavior of foraging bat. Cuckoo Search is based on the brooding parasitism of the cuckoo species. Among these algorithms, Firefly algorithm is considered to be better since it is having minimum running time and better success rate. So it can be used for large scale application efficiently [13].

Ant Colony Optimization have better success rate but running time is medium and it require more CPU time as compared to others.

Table 1. Algorithm Comparison

\begin{tabular}{|l|l|l|l|l|}
\hline $\begin{array}{l}\text { Name Of } \\
\text { Algorithm }\end{array}$ & $\begin{array}{l}\text { Running } \\
\text { Time }\end{array}$ & $\begin{array}{l}\text { Success } \\
\text { Rate }\end{array}$ & Classification & $\begin{array}{l}\text { Algorithm } \\
\text { Basis }\end{array}$ \\
\hline $\begin{array}{l}\text { Firefly } \\
\text { Algorithm }\end{array}$ & Minimum & Better & $\begin{array}{l}\text { Population } \\
\text { Based, } \\
\text { Attraction } \\
\text { Based } \\
\text { Algorithm }\end{array}$ & $\begin{array}{l}\text { Flashing } \\
\text { Behavior of } \\
\text { Swarming } \\
\text { Firefly }\end{array}$ \\
\hline $\begin{array}{l}\text { Ant Colony } \\
\text { Optimization }\end{array}$ & Medium & Better & $\begin{array}{l}\text { Population } \\
\text { Based } \\
\text { Algorithm }\end{array}$ & $\begin{array}{l}\text { Interaction } \\
\text { of } \\
\text { Species Ant }\end{array}$ \\
\hline $\begin{array}{l}\text { Bat } \\
\text { Algorithm }\end{array}$ & Medium & Intermediate & $\begin{array}{l}\text { Population } \\
\text { Based } \\
\text { Algorithm }\end{array}$ & $\begin{array}{l}\text { Echolocation } \\
\text { of Foraging } \\
\text { Bats }\end{array}$ \\
\hline $\begin{array}{l}\text { Cuckoo } \\
\text { Search }\end{array}$ & Maximum & Low & $\begin{array}{l}\text { Equation } \\
\text { Based } \\
\text { Algorithm }\end{array}$ & $\begin{array}{l}\text { Brooding } \\
\text { Parasitism of } \\
\text { Cuckoo Bird }\end{array}$ \\
\hline
\end{tabular}

\section{CONCLUSION}

From the comparison of firefly, bat, cuckoo and ant colony optimization algorithms, it is clear that firefly algorithm is the most efficient one. It is the most powerful as well as favorable optimization tool. Time complexity is of firefly algorithm is lesser than other algorithms. So the algorithm is efficiently used for Job Shop Scheduling Problem (JSSP). Cuckoo Search need more time. Its main application is in training the network for the removal of noise.

Bat algorithm finds its application in classification of gene expression data. Ant colony optimization has better running time and is mainly used in scheduling problems and travelling salesman problem.

\section{REFERENCES}

[1] M. Dorigo and G. Di Caro, "New ideas in optimization", 1999.

[2] Tang Rui, Simon Fong, Xin-She Yang and Suash Deb, "Nature-inspired Clustering Algorithms for Web Intelligence Data," pp. 978-0-7695-4880-7 vol. 12, 2012.

[3] Sankalp Arora, Satvir Singh, "A Conceptual Comparison of Firefly Algorithm,Bat Algorithm and Cuckoo Search,"pp. 978-1-4799-1375, 2013.

[4] Kuang Xiangling and Huang Guangqiu,“ An Optimization Algorithm Based on Ant Colony Algorithm," 2012.

[5] Iztok Fister Jr., Xin-She Yang, Janez Brest and Dusan Fister," A Brief Review of Nature-Inspired Algorithms for Optimization," Elektrotehniski vestnik 80(3):17,English Edition, 2013.

[6] Rui Tang, Simon Fong, Xin-She Yang, Suash Deb," Integrating Nature-inspired Optimization Algorithms to K-means Clustering,” IEEE Trans.,pp. 978-1-4673-2430, 2012. 
[7] X.-S. Yang,"Nature-inspired metaheuristic algorithms", Luniver Press, 2011

[8] Z. W. Geem, J. H. Kim, and G. Loganathan, "A new heuristic optimization algorithm: harmony search," Simulation, vol. 76, no. 2, pp. 60-68, 2001.

[9] A. Kaveh and S. Talatahari, "A novel heuristic optimization method: charged system search," Acta Mechanica, vol. 213, no. 3-4, pp.267-289, 2010.

[10] Homayun Afrabandpey, Meysam Ghaffari, Abdolreza Mirzaei, Mehran Safayani,"A Novel Bat Algorithm Based on Chaos for Optimization Tasks,"pp. 4799-3351, 2014.
[11] Kunja Bihari Swain, S.S Swalanki and Asim Kumar mahakula, "Bio Inspired Cuckoo Search Algorithm Based Neural Network and its Application to Noise Cancellation,” pp.4799-2866, 2014.

[12] Siddharth Agarwal, Amrit Pal Singh, Nitin Anand, "Evaluation performance study of firefly algorithm, particle-swarm optimization and artificial bee colony algorithm for non-linear mathematical optimization function," ICCCNT, 2013.

[13] Roberto A. Vazquez, "Training Spiking Neural Models using Cuckoo Search Algorithm," pp. 978-1-4244-78354, 2011. 